\title{
Deutsche Initiative für Netzwerkinformation (DINI): Gremien neu besetzt
}

\section{Die Deutsche Initiative für Netzwerkinformation (DINI) hat einen neuen Vorstand und einen neuen Hauptausschuss gewählt}

https://doi.org/10.1515/bd-2021-0007

Im Rahmen der 21. Jahrestagung haben die Mitglieder der Deutschen Initiative für Netzwerkinformation (DINI) einen neuen Vorstand und einen neuen Hauptausschuss für die Amtszeit 2020-2022 gewählt.

Weiterhin wird Dr. Helge Steenweg (IZUS / Universitätsbibliothek Stuttgart) den Dachverband von Bibliotheken, Medienzentren und Rechenzentren an Hochschulen und außeruniversitären Forschungseinrichtungen als Vorstandsvorsitzender leiten. Stellvertretender DINI-Vorstandsvorsitzender ist Boguslaw Malys (Brandenburgische Technische Universität Cottbus-Senftenberg).

Der Vorstand setzt sich wie folgt zusammen:

- Prof. Dr. Ursula Arning, ZB MED - Informationszentrum Lebenswissenschaften

- Thomas Bieker, Hochschule Ruhr West

- Barbara Knorn, Universitätsbibliothek Bielefeld

- Dr. Peter Leinen, Deutsche Nationalbibliothek

- Boguslaw Malys, BTU Cottbus-Senftenberg

- Heinz Pampel, Helmholtz-Gemeinschaft

- Uwe Pirr, Humboldt-Universität zu Berlin, Computer- und Medienservice

- Dr. Helge Steenweg, IZUS / Universitätsbibliothek Stuttgart

Auch wurde der Hauptausschuss neu gewählt. Weiter konnte der Vorstand neue Mitglieder für den Beirat gewinnen. Eine Liste aller Mitglieder der drei Gremien findet sich auf der DINI-Webseite https://dini.de/dini/gremien.

Die virtuelle DINI-Jahrestagung stand vom 29. und 30. September 2020 unter dem Motto „UX 2020 - Fokus User Experience“ und widmete sich den bisherigen Erfahrungen im Umgang mit der Corona-Pandemie auf Forschung und Lehre. U. a. berichtete Professorin Dr. Eva Inés Obergfell, Vizepräsidentin für Lehre an der Humboldt-Universität zu Berlin, über Studieren und Lehren in Zeiten von Corona und Professor Dr. York Sure-Vetter, Direktor der Nationalen Forschungs- 
dateninfrastruktur, über den Stand der Nationalen Forschungsdateninfrastruktur (NFDI), deren erste Konsortien seit Oktober 2020 von Bund und Ländern finanziert werden.

Im Rahmen der DINI-Jahrestagung ging auch ein gemeinsames Blog der Arbeitsgruppen Elektronisches Publizieren und Forschungsinformationssysteme online. Das Blog widmet sich dem Open Access-Publizieren, der Forschungsberichterstattung und weiteren Aspekten rund um die digitale Wissenschaftskommunikation und das Informationsmanagement an Hochschulen und außeruniversitären Einrichtungen. Das Blog findet sich unter https://blogs.tib.eu/wp/ dini-ag-blog.

Neues aus DINI gibt es auch zum Thema Forschungsdatenmanagement. Eine neue Ausgabe der Open Access-Zeitschrift „Bausteine Forschungsdatenmanagement“ informiert über Praktiken im Umgang mit digitalen Forschungsdaten. Die Zeitschrift kann unter https://bausteine-fdm.de abgerufen werden.

Für das Jahr 2020 stehen noch zwei DINI-Veranstaltungen an:

- Unter dem Titel „Fünf Jahre Kerndatensatz Forschung im deutschen Wissenschaftssystem“ organisieren vom 9. bis 10. November 2020 das Deutsche Zentrum für Hochschul- und Wissenschaftsforschung und die DINIArbeitsgruppe „Forschungsinformationssysteme“ eine öffentlich zugängliche virtuelle Veranstaltung zum Thema Kerndatensatz Forschung. Weitere Informationen unter https://dini.de/veranstaltungen/workshops/fuenf-jahrekerndatensatz-forschung-im-deutschen-wissenschaftssystem-umsetzungentwicklungen-und-perspektiven.

- Die DINI-Arbeitsgruppe Forschungsdaten befasst sich in der virtuellen Veranstaltung „Data Stewardship im Forschungsdatenmanagement - Was ist das? Rollen, Aufgabenprofile, Einsatzgebiete“ befasst sich vom 16. bis 17. November 2020 in Kooperation mit der Universität zu Köln und dem ZB MED - Informationszentrum Lebenswissenschaften mit der Personalentwicklung im Bereich des Forschungsdatenmanagement. Weitere Informationen unter https://dini.de/veranstaltungen/workshops/data-stewardship-imforschungsdatenmanagement-was-ist-das-rollen-aufgabenprofile-einsatzge biete.

\section{Über die Deutsche Initiative für Netzwerkinformation (DINI)}

Die Deutsche Initiative für Netzwerkinformation (DINI) e. V. ist der überregionale Zusammenschluss von wissenschaftlichen Bibliotheken, Medienzentren, Rechenzentren und Fachgesellschaften in Forschung und Lehre in Deutschland. DINI ist Partnerin von Hochschulen und außeruniversitären Forschungseinrichtungen bei der Weiterentwicklung der Informationsinfrastrukturen. Weitere Informationen finden Sie unter https://dini.de. 


\section{Kontakt:}

Claudia Walther / DINI e.V. Geschäftsstelle

c/o Niedersächsische Staats- und Universitätsbibliothek Göttingen

37073 Göttingen | Platz der Göttinger Sieben 1

Tel.: +4955139-33857 |E-Mail: claudia.walther@sub.uni-goettingen.de, gs@dini.de 